\title{
Functional brain mapping using positron emission tomography scanning in preoperative neurosurgical planning for pediatric brain tumors
}

\author{
Allen M. Kaplan, M.D., Daniel J. Bandy, M.S., Kim H. Manwaring, M.D., \\ Kewei Chen, Ph.D., Michael A. Lawson, M.D., S. David Moss, M.D., \\ Johnny D. Duncan, D.O., David L. Wodrich, Ph.D., James A. Schnur, M.D., \\ AND ERIC M. REIMAN, M.D.
}

Departments of Child Neurology, Neurosurgery, Psychology, Radiology, and the Positron Emission Tomography Center, Phoenix Children's Hospital/Good Samaritan Regional Medical Center, Phoenix Arizona; Department of Pediatrics, Maricopa Medical Center, Phoenix, Arizona; and Departments of Pediatrics, Psychiatry and Neurosurgery, University of Arizona School of Medicine, Tucson, Arizona

Object. The purpose of this report is to demonstrate the value of functional brain mapping using the positron emission tomography (PET) method for preoperative neurosurgical planning in children with brain tumors. Brain maps were used to characterize the relationship between potentially resectable tumors and functionally eloquent brain areas.

Methods. Five children, ranging in age from 3 to 13 years, with hemispheric brain tumors adjacent to eloquent cortex were studied. Magnetic resonance (MR) imaging was used to identify the brain tumors; PET imaging after injection of $\left[{ }^{18} \mathrm{~F}\right]$ fluorodeoxyglucose (FDG), $\left[{ }^{11} \mathrm{C}\right] \mathrm{L}$-methionine (CMET), or a combination of the two was performed to grade the tumors; and $\left.\mathrm{a}^{15} \mathrm{O}\right] \mathrm{H}_{2} \mathrm{O}$ uptake study was used to characterize the anatomical relationships of the tumors to functional cortex. The cortical activation maps were obtained during control periods and during behavioral tasks and were used to document motor, visual, and speech and language organizational areas. Wada tests were performed in two patients. Language and speech activation was concordant with the results of Wada testing.

Conclusions. Functional brain mapping using PET scans and coregistered MR images provided the neurosurgeon with precise definitions of structural and functional cortical areas; this altered surgical management in some cases and/or was used to predict outcome. The combination of PET imaging with FDG and/or CMET and measurements an $\left[{ }^{5} \mathrm{O}\right]$ was of $\left.{ }^{15} \mathrm{O}\right]$ water uptake was useful in characterizing and grading tumors and instrumental in achieving effective neurosurgical planning. Postoperative results in the five cases suggest that preoperative functional brain mapping has the potential to improve outcome by defining a surgical plan to maximize resection and minimize the risk of neurological sequelae.

KEY WORDS - functional brain mapping - pediatric brain neoplasm •

positron emission tomography $\bullet$ children

The localization or mapping of cortical functions by noninvasive techniques is rapidly becoming a useful too in the preoperative assessment of neurosurgical procedures for epilepsy, vascular malformations, and brain tumor resections. ${ }^{3,6,9,13,15}$ Functional brain mapping using positron emission tomography (PET) to measure regional cerebral blood flow (rCBF) has characterized regions of the central nervous system involved in visual, sensory, motor, auditory, memory, attention, and language functions. $7,8,16,17,20,21$ Although used in adult patients more frequently, these techniques can be applied to the pediatric age group. The localization and identification of functional cortical areas in patients with brain tumors provide the neurosurgeon with the ability to achieve aggressive resections with preservation of neurological function. Functional maps may also eliminate or modify the use of inva- sive surgical procedures, reducing the risks of neurological impairment and potentially defining specific areas for adjuvant therapies.

Before scheduled neurosurgical operations, we studied five children who harbored various brain tumors by using coregistered magnetic resonance (MR) imaging and PET images, including functional brain mapping techniques. In all cases the PET data including the functional maps were instrumental in grading or metabolically characterizing the tumors and in altering surgical management and/or predicting outcome. Our experience with PET mapping demonstrates this method to be a clinically practical, welltolerated, and reliable technique even when used in young children. The clinical application and usefulness of PET mapping techniques for surgical planning in these brain tumor cases will be reviewed. 
TABLE 1

Summary of clinical data*

\begin{tabular}{|c|c|c|c|c|c|c|c|c|}
\hline $\begin{array}{l}\text { Case } \\
\text { No. }\end{array}$ & $\begin{array}{c}\text { Age } \\
\text { (yrs), } \\
\text { Sex }\end{array}$ & Presentation & Tumor Location & Pathological Diagnosis & $\begin{array}{c}\text { MR } \\
\text { Appearance }\end{array}$ & $\begin{array}{l}\text { PET Findings } \\
\text { FDG/CMET }\end{array}$ & $\begin{array}{c}\text { Wada } \\
\text { Test Result }\end{array}$ & $\begin{array}{c}\text { Neuro- } \\
\text { surgical } \\
\text { Procedure }\end{array}$ \\
\hline 1 & $12, \mathrm{M}$ & $\begin{array}{l}\text { generalized } \\
\text { seizures }\end{array}$ & It thalamic/basal ganglia & pilocytic astrocytoma & enhancing & $\begin{array}{l}\text { hypometabolic/increased } \\
\text { metabolic uptake }\end{array}$ & $\begin{array}{l}\text { rt hemispheric } \\
\text { dominance }\end{array}$ & GTR \\
\hline 2 & $13, \mathrm{~F}$ & partial seizures & rt temporal lobe & DNT & nonenhancing & $\begin{array}{l}\text { hypometabolic/ } \\
\text { hypometabolic }\end{array}$ & $\begin{array}{l}\text { rt hemispheric } \\
\text { dominance }\end{array}$ & GTR \\
\hline 3 & $12, \mathrm{~F}$ & partial seizures & It insula & DNT & nonenhancing & $\begin{array}{l}\text { hypometabolic/ } \\
\text { hypometabolic }\end{array}$ & NA & GTR \\
\hline 4 & $10, \mathrm{M}$ & lt hemiparesis & rt frontotemporal lobe & pilocytic astrocytoma & enhancing & $\begin{array}{l}\text { hypometabolic/increased } \\
\text { metabolic uptake }\end{array}$ & NA & GTR \\
\hline 5 & $3, \mathrm{M}$ & partial seizures & It parietooccipital lobe & oligodendroglioma & enhancing & NA & NA & NTR \\
\hline
\end{tabular}

* GTR $=$ gross-total resection; NA = not available; NTR = near-total resection

\section{Clinical Material and Methods}

\section{Patient Population}

The study included five children or adolescents ranging in age from 3 to 13 years. Coregistered MR images and PET scans obtained using $\left[{ }^{18} \mathrm{~F}\right]$ fluorodeoxyglucose (FDG) in four cases and $\left[{ }^{11} \mathrm{C}\right] \mathrm{L}$-methionine (CMET) in three cases were used to establish the metabolic character and grade of the brain lesions. Functional brain mapping by using $\left[{ }^{15} \mathrm{O}\right] \mathrm{H}_{2} \mathrm{O}$ uptake was performed in all cases to map eloquent brain areas as part of the preoperative evaluation for brain tumor resection. Clinical data, including patient age and gender, presentation, tumor location, pathological typing, the results of MR and PET imaging studies and Wada tests, and the outcomes of neurosurgery were tabulated (Table 1).

\section{Magnetic Resonance Imaging}

Magnetic resonance images were obtained using a 1.5tesla system (Signa; General Electric, Milwaukee, WI). In four of the five patients, a $\mathrm{T}_{1}$-weighted three-dimensional spoiled-gradient sequence $(192 \times 256$ matrix, TR 33 $\mathrm{msec}$, TE $5 / 30 \mathrm{msec}$, field of view [FOV] $24 \mathrm{~cm}$ ) that produces 1.5-mm-thick contiguous images was acquired and used for subsequent coregistration with the PET data. In the other patient, technical limitations prevented us from using this sequence and $\mathrm{T}_{2}$-weighted fast-spin echo images $(192 \times 256$ matrix, TR $3750 \mathrm{msec}$, TE $18 / 126 \mathrm{msec}$, FOV $22 \mathrm{~cm}$ ) with a $5-\mathrm{mm}$ slice thickness and a $2.5-\mathrm{mm}$ interslice space were used for coregistration.

\section{Positron Emission Tomography Imaging}

Positron emission tomography scans (ECAT 951/31 scanner; CTI/Siemens, Knoxville, TN) were used to: 1 ) acquire repeated measurements of $\mathrm{CBF}$ during well-characterized behavioral and baseline tasks; 2) image methionine metabolism (Cases 1, 3, and 4); and 3) image the cerebral metabolic rate for glucose (Cases $1-4)$. The scanner produces 31 transaxial slices with a center-to-center slice separation of $3.4 \mathrm{~mm}$, a $10.8-\mathrm{cm}$ axial FOV, an in-plane resolution of 5.8 to $7.7 \mathrm{~mm}$ full width at half maximum (FWHM) in the center of the FOV, and an axial resolution of 5 to $7.1 \mathrm{~mm}$ FWHM. Attenuation correction was performed using either a standard ellipse-fitting method with an attenuation coefficient of $0.95 \mathrm{~cm}^{-1}$ or a measured attenuation correction using an external ${ }^{68} \mathrm{Ge}$ ring source with 30 minutes of data acquisition. Images were reconstructed using a Hanning filter at a cutoff frequency of

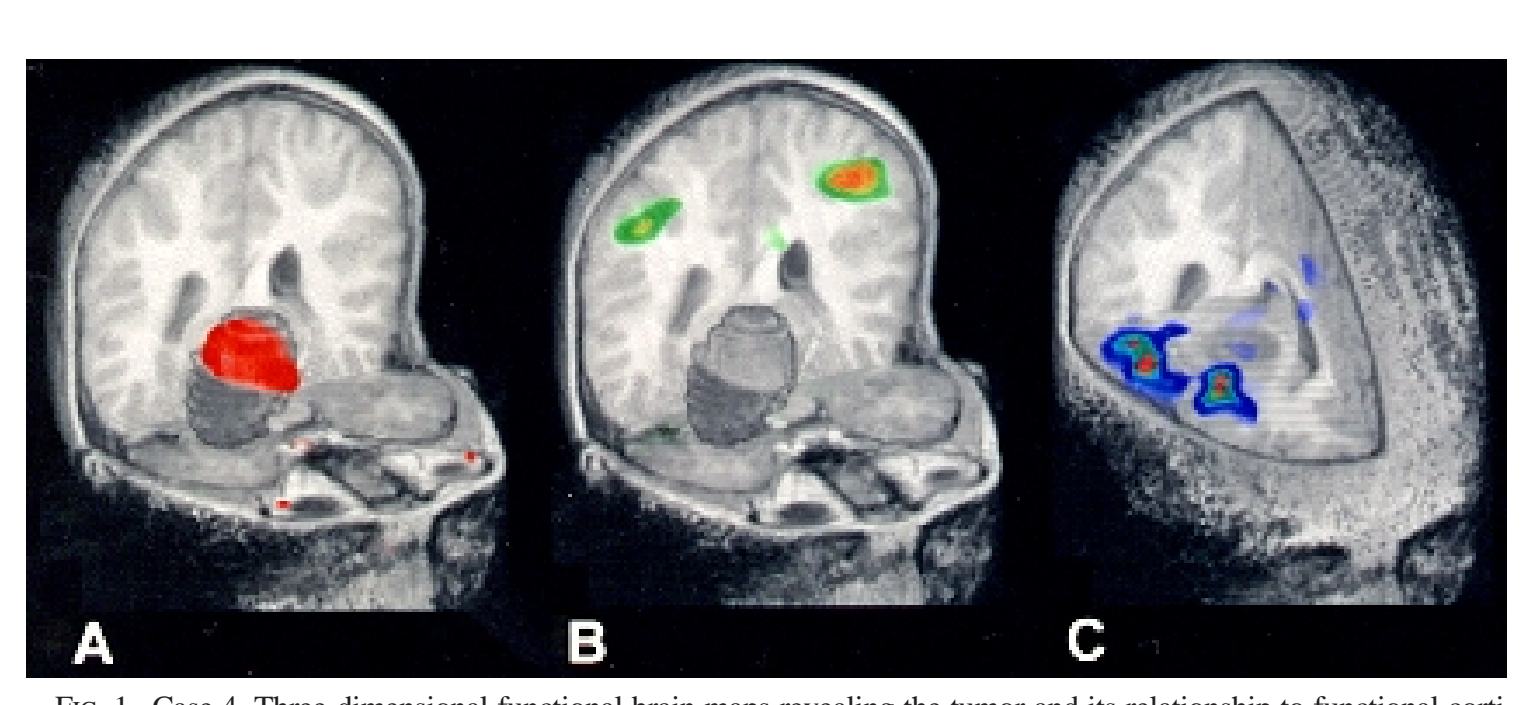

FIG. 1. Case 4. Three-dimensional functional brain maps revealing the tumor and its relationship to functional cortical areas: metabolic activity (CMET scan; A), motor activation (B), and speech and language activation (C). 
Functional brain mapping for pediatric brain tumor surgery

0.4 cycles/pixel, yielding an in-plane resolution of 9.5 $\mathrm{mm}$ FWHM.

The patients undergoing FDG and CMET PET imaging were instructed to fast for at least 4 hours before the procedure. Baseline plasma glucose levels were measured and determined to be less than $120 \mathrm{mg} / \mathrm{dl}$ in all patients. Intravenous infusion of 5 to $10 \mathrm{mCi}$ of CMET was administered. A 60-minute dynamic imaging sequence was initiated at the time of injection. Image frames obtained in 10 to 60 minutes were summed and used for subsequent coregistration. A second infusion of $0.014 \mathrm{mCi} / \mathrm{kg}$ body weight of FDG was administered. The patients were instructed to rest quietly with their eyes closed for $30 \mathrm{~min}$ utes, at which time they were positioned in the PET scanner and a 30-minute static scan was acquired.

Functional Brain Mapping Methods (Paradigms)

Intravenous bolus injections of 25 to $50 \mathrm{mCi}$ of $\left[{ }^{15} \mathrm{O}\right]$ $\mathrm{H}_{2} \mathrm{O}$ were given and 60 -second static scans were initiated as the tracer arrived at the brain, as noted by the online count monitor. Serial scans 10 to 15 minutes apart were obtained as the patient was observed for relevant behavior and baseline paradigms. For language activation, the patient was instructed to read the name of a common animal as it appeared on a computer monitor suspended in front of the patient who was positioned in the PET scanner. The patient was instructed to say the name of the animal aloud and then answer "yes" or "no" to indicate if the animal was dangerous. Each animal name was presented for 1.5 seconds with a 1.5 -second delay between presentation. The tasks were designed to identify brain areas previously implicated in recognition of written-spoken work (visual association areas in the occipital cortex and auditory association areas in the temporal lobe, respectively), articulation (dominant inferior frontal cortex), and semantic associations (anterior cingulate gyrus). In the case of a 3year-old patient, the paradigm was modified, with an assistant standing behind the scanner gantry and verbally reading the animal names. The patient was instructed to repeat the animal name and say "yes" or "no" as to whether the animal was big or small. For motor activation, the patients were instructed to open and close their hands repeatedly.

\section{Image Analysis/Coregistration Imaging}

Automated algorithms were used to align each patient's PET images, normalize them for variations in whole brain measurements, compute images of mean increases in $\mathrm{rCBF}$ related to behavioral tasks, and superimpose the images of mean changes in rCBF, methionine, and FDG uptake onto the MR images. ${ }^{23}$ Color-coded maps for recording mean changes in $\mathrm{rCBF}$ were manually adjusted and visually inspected to distinguish state-dependent changes in $\mathrm{rCBF}$ from noise. Three-dimensional functional maps demonstrating the relationship of tumor to eloquent cortical areas were produced (Fig. 1).

\section{Wada Testing}

The intracarotid Amytal (Wada) test was accomplished using the standard techniques to lateralize speech described by Wada and Rasmussen. ${ }^{22}$ The patient was famil-

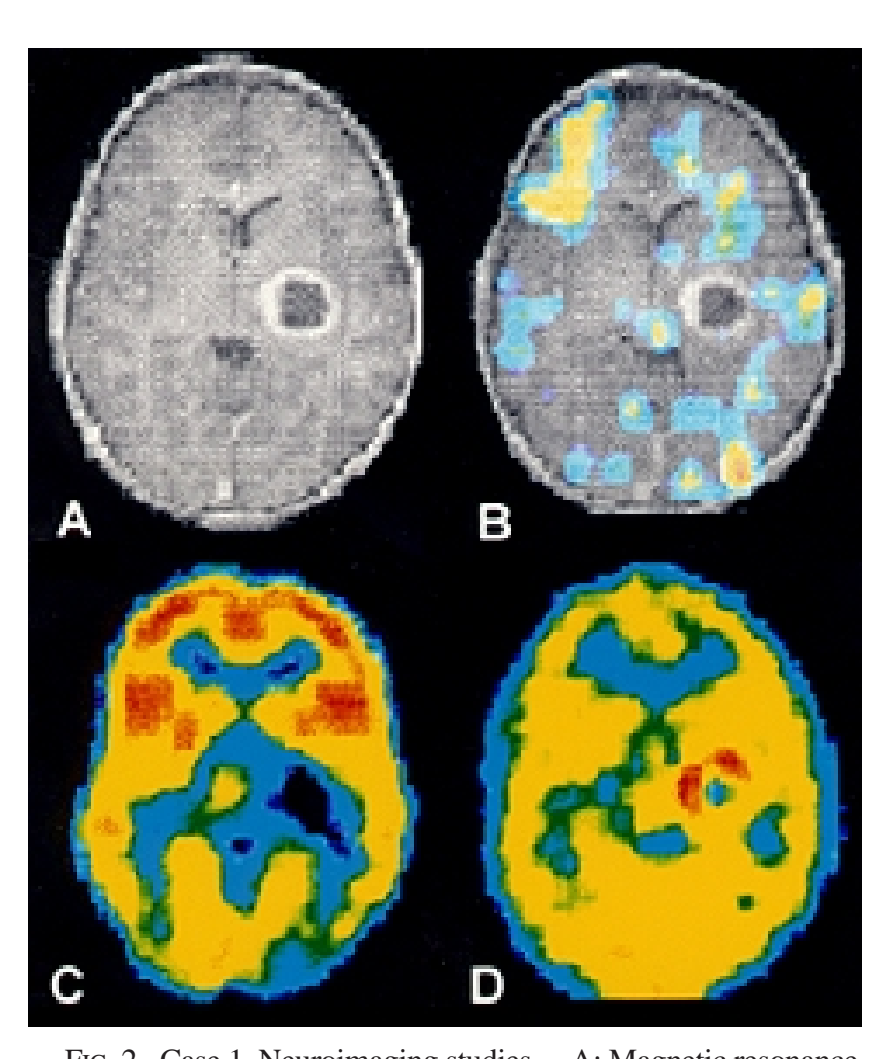

FIG. 2. Case 1. Neuroimaging studies. A: Magnetic resonance imaging revealing a left hemispheric cystic tumor. B: Functional brain map demonstrating right hemispheric speech and language areas and motor function lateral to the tumor. C: An FDG PET scan demonstrating hypometabolism. D: A CMET PET scan demonstrating increased peripheral metabolism.

iarized with the testing format before the Amytal study. The pretest assessment of language dominance consisted of counting; naming pictured objects, shapes, and colors; repeating a short phrase; and following a simple direction with memory assessed by the patient's ability to recall or recognize the test items after medication effects had dissipated.

\section{Results}

The cortical mapping studies in four patients in the current series were instrumental in altering the management of, or surgical approach to, the tumor and, in one patient, the functional maps predicted outcome.

In Case 1, MR imaging revealed a contrast-enhancing cystic lesion in the deep white matter of the left hemisphere. Positron emission tomography scanning of the tumor demonstrated hypometabolism with addition of FDG and increased metabolic activity with addition of CMET, suggesting a low-grade pathological entity. Functional brain mapping revealed dominant speech and language functions in the right hemisphere with motor function lateral to the tumor (Fig. 2). An image-guided endoscopic procedure, which included biopsy, confirmed the presence of a pilocytic astrocytoma. On the basis of the histological diagnosis and the unexpected laterality of language demonstrated by PET scanning, an aggressive surgical procedure with gross-total resection of the tumor was per- 
A. M. Kaplan, et al.

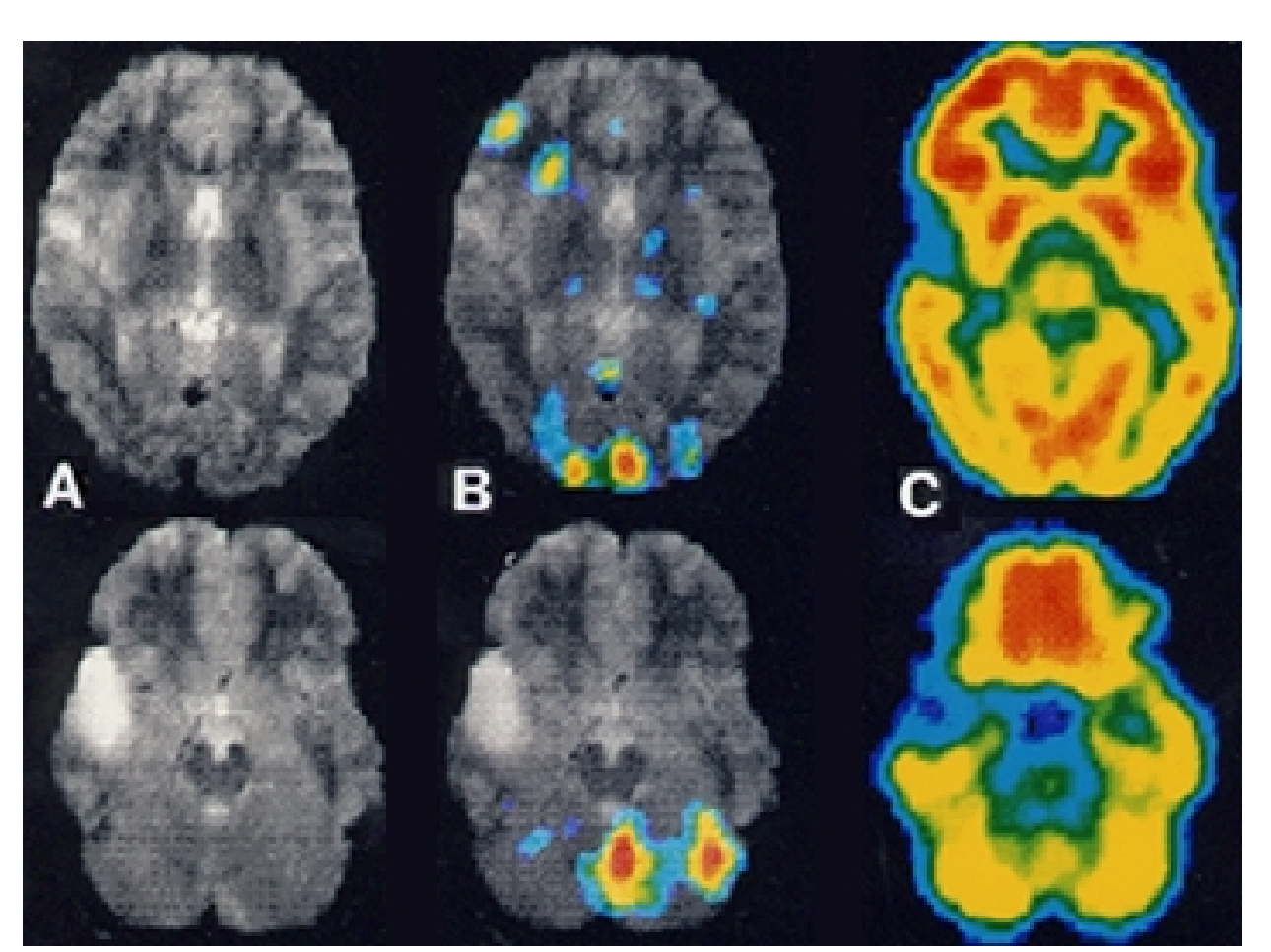

FIG. 3. Case 2. Neuroimaging studies. A: Magnetic resonance images revealing a right temporal lobe tumor. B: Functional brain maps demonstrating speech and language areas adjacent to the tumor. C: An FDG PET scan revealing hypometabolism.

formed. There were no significant neurological sequelae, as predicted by the PET data.

The patient in Case 2 harbored a nonenhancing, lowdensity right temporal lobe lesion. Positron emission tomography scans obtained using FDG and CMET revealed no metabolic activity, suggesting a low-grade tumor or dysplastic process. Functional brain mapping demonstrated right hemispheric expressive and receptive speech organizational areas adjacent to the neoplastic lesion (Fig 3 ). In view of the functional mapping data, a craniotomy performed via a lateral and inferior approach allowed cortical entry outside the language and speech areas and gross-total resection of the tumor. This approach also allowed preservation of the visual pathways. The final pathological diagnosis was a dysembryoplastic neuroepithelial tumor (DNT). No speech, motor, or visual defects were documented during the postoperative course.

In the patient in Case 3 a nonenhancing $2-\mathrm{cm}$ lesion within the left insular region was revealed on MR images. Positron emission tomography scans after FDG or CMET injection demonstrated hypometabolism suggesting a low-grade tumor or dysplasia. Functional brain maps showed left-sided speech and language dominance anterior to the tumor (Fig. 4). A left posterior temporal approach was chosen to avoid eloquent cortical areas mapped by functional studies. The gyrus selected for corticotomy was identified by matching the anatomy on the coregistered PET-MR sagittal images to the cortex visualized at surgery, allowing gross-total resection of the neoplasm. The final pathological diagnosis was a DNT. The patient's postoperative course was uncomplicated. There were no significant speech or language problems identified.
The patient in Case 4 underwent MR imaging, which revealed an enhancing lesion with a large cystic component that lay deep within the right hemisphere adjacent to the anterior limb of the internal capsule and displaced the basal ganglia medial and inferior to the tumor. No significant metabolic activity was demonstrated using FDG PET scanning. The CMET study, however, showed significant uptake in the temporal cortex and basal ganglia, suggesting a low-grade glioma. Functional brain mapping demonstrated displacement of both receptive and expressive speech areas lateral and anterior to the neoplasm in the right hemisphere, with motor and speech areas superior to the neoplasm (Fig. 5). In view of the functional findings, a craniotomy via an inferior approach, which entered the posterior middle temporal gyrus and avoided speech areas, was performed. Subtotal resection was completed and the final pathological diagnosis was a pilocytic astrocytoma. Because of the benign pathological condition, a second-look procedure was performed with gross-total resection of the tumor. A transient postoperative aphasia and hemiparesis resolved before the patient was discharged from the hospital.

In Case 5, the youngest patient in this series at 3 years of age, MR imaging revealed a large enhancing lesion involving the left temporal, parietal, and occipital areas. The tumor was inhomogeneous, being composed of both cystic and solid components. Functional brain mapping in which a modified paradigm was used documented bilateral speech representation with displacement of speech and motor function in the left hemisphere (Fig. 6). The patient underwent a craniotomy, with near-total resection of the neoplasm, in which the surgeon attempted to leave speech 


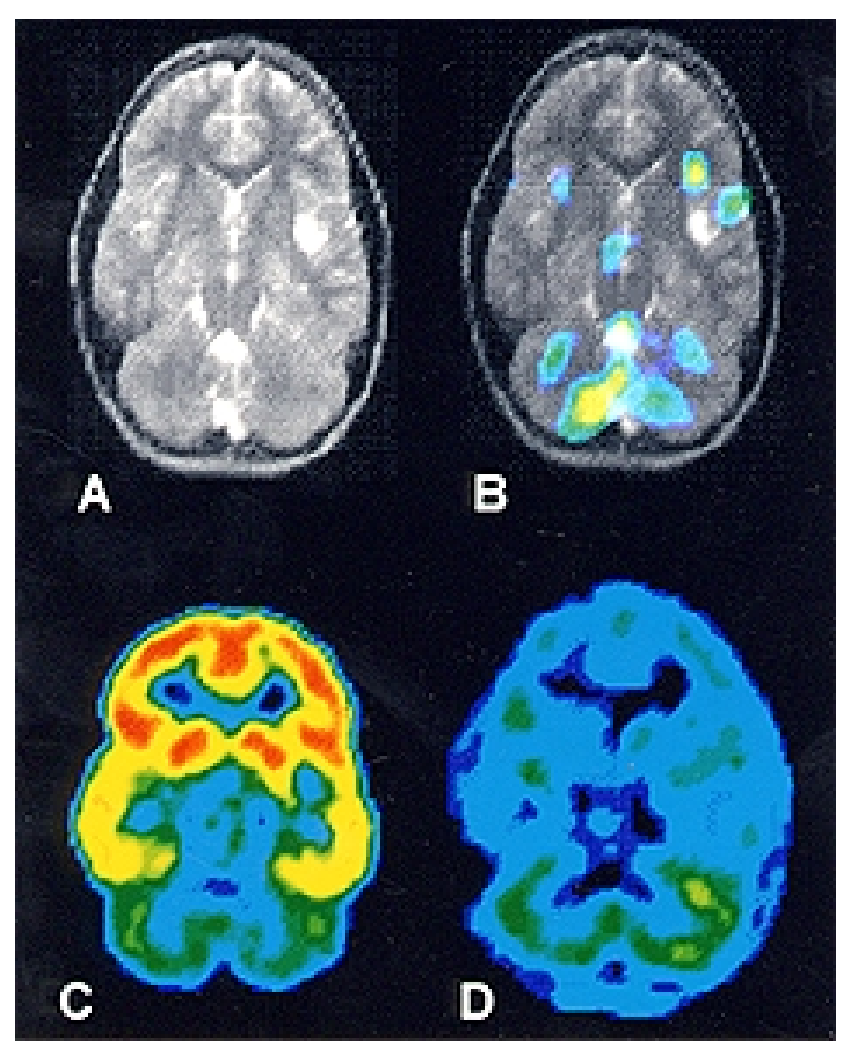

FIg. 4. Case 3. Neuroimaging studies. A: Magnetic resonance image revealing a left hemispheric nonenhancing tumor. B Functional brain map demonstrating speech and language areas anterior to the tumor. C: Hypometabolic FDG PET findings. D: Hypometabolic CMET PET findings.

areas virtually surrounding the lesion intact. The final pathological diagnosis was a low-grade oligodendroglioma. The child's postoperative course was benign with no neurological deficits and a follow-up neuroimaging study demonstrated no progressive disease.

Although performed in only two cases (Cases 1 and 2), the results of Wada tests documented right hemispheric dominance in both patients and were concordant with PET functional studies.

The FDG and CMET PET studies documented decreased metabolic activity in both patients who had DNTs. The PET scans demonstrated reduced FDG metabolic activity with increased CMET activity in both pilocytic tumors. Positron emission tomography scans were not obtained in Case 5.

\section{Discussion}

In the past, localization of higher cortical functions has relied on anatomical studies, which require both clinical and pathological correlation. Recent advances in neuroimaging with computerized tomography scanning and MR imaging have dramatically improved localization and characterization of structural and anatomical lesions within the central nervous system. Direct cortical stimulation mapping during brain tumor surgery has allowed the identification of essential language and motor areas, thereby

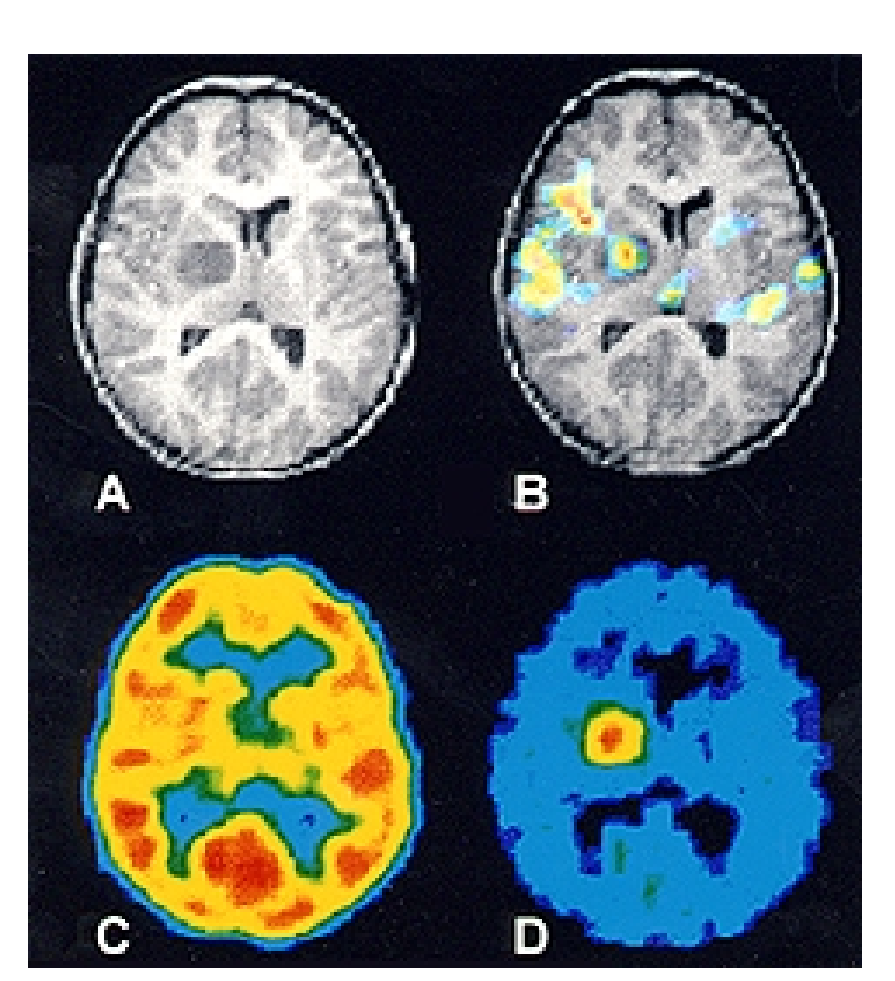

FIG. 5. Case 4. Neuroimaging studies. A: Magnetic resonance image revealing a deep right temporal/basal ganglia neoplasm. $B$ : Functional brain map demonstrating speech and language organizational areas adjacent to the tumor. The increased metabolic activation blood flow shows actual functional areas within the tumor. C: An FDG PET scan demonstrating hypometabolism. D: A CMET PET scan demonstrating hypermetabolism.

allowing the surgeon to maximize tumor resection and minimize speech and language deficits. ${ }^{10}$ Patients, however, must remain awake during the craniotomy and electrical stimulation of the cortex. With further improvements in computer software, imaging and mapping of cerebral function by using noninvasive PET activation and functional MR (fMR) imaging techniques are now achievable. The ability to identify various functional cortical areas in a patient, including receptive and expressive language, motor, and visual functions, helps the surgeon understand the combined anatomical and functional configuration of the brain lesion and adjacent areas, which improves the approach to surgery and overall case management.

Utilization of PET activation studies in the preoperative management of cerebral lesions was introduced by LeBlanc and Meyer ${ }^{15}$ in 1990. Functional PET scanning precisely localized a structural brain lesion to the precentral gyrus, which was validated by intraoperative cortical mapping. A second study, ${ }^{16}$ in which activation of speech and language areas was used, improved the management of a vascular lesion and demonstrated that the PET technique was useful to the neurosurgeon in decisions regarding direct surgery, embolization, or radiosurgery for vascular malformations.

Fried, et al., ${ }^{8}$ compared fMR and PET imaging of rolandic and visual cortices and concluded that both techniques could be used to achieve preoperative brain mapping. Atlas, et al., ${ }^{2}$ used fMR imaging to localize areas of cortical

Neurosurg. Focus / Volume 8 / February, 2000 


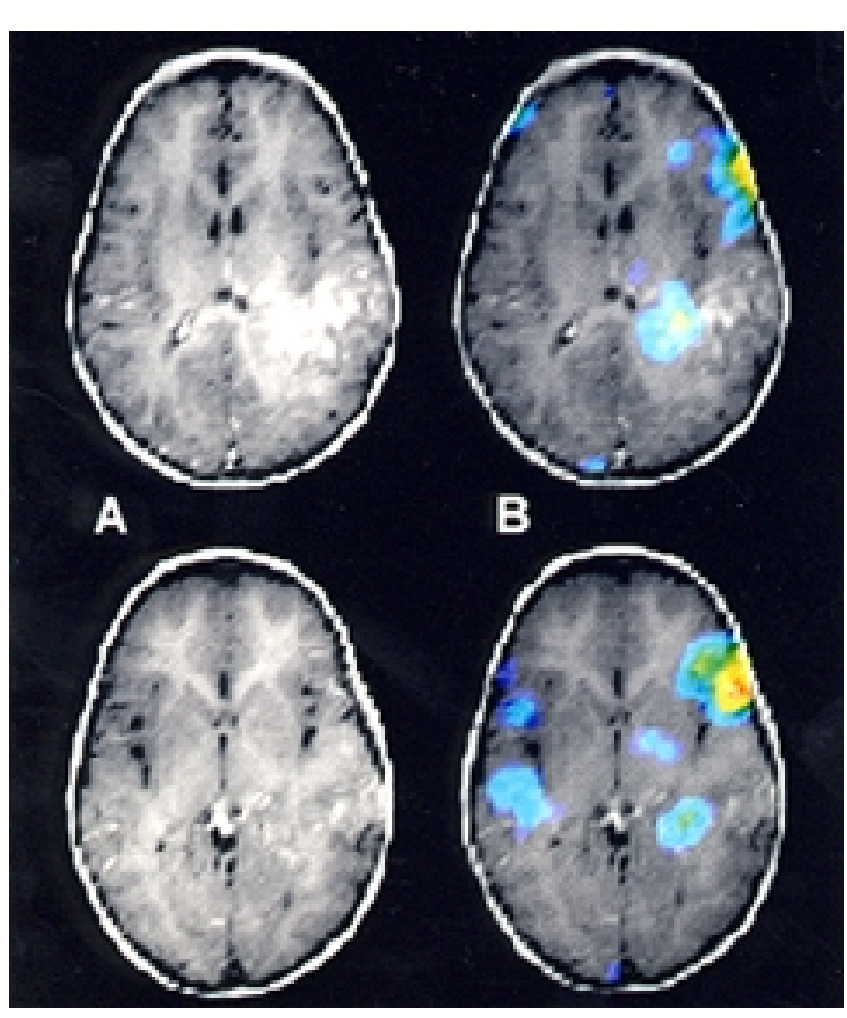

FIG. 6. Case 5. Neuroimaging studies. A: Magnetic resonance images revealing extensive inhomogeneous tumor. B: Functional brain maps demonstrating bilateral speech and language areas with displacement of expressive speech and motor function in the left hemisphere

function for treatment planning in patients undergoing glioma surgery. Their data indicated that functioning cortex within or adjacent to tumor margins could be identified, providing pretreatment maps for both surgery and radiation therapy. Mueller and colleagues ${ }^{18}$ confirmed that the fMR imaging technique was useful in determining the risks of postoperative motor deficits in patients with frontoparietal tumors. Resections exceeding $2 \mathrm{~cm}$ between the margins of the tumor and adjacent motor areas resulted in no decline in patient motor function.

The apparent advantages of PET scanning over fMR imaging include less claustrophobic anxiety for the patient, tumor grading capability, and mapping in a single session. Positron emission tomography scanning can be performed easily in children without significant disruption of functional maps by movement artifacts. The disadvantages include radiation exposure, albeit at a low level, and a lower spatial resolution.

With the development of functional imaging and cortical stimulation studies, the organization of speech and language has evolved from the original concepts of decoding in the posterior temporal cortex (Wernicke's area) to motor expression in the posterior inferior frontal lobe (Broca's area). Language appears to be composed of several essential areas localized to the frontal and temporoparietal cortex, as well as more widely dispersed neurons activated in parallel. ${ }^{19}$ Subcortical input to speech and language function also has been documented. The results of cortical mapping studies in this report can be used to confirm both the anatomical and functional variances of language organization. In three of our cases right hemispheric speech and language organizational areas were demonstrated. In two cases, the functional language areas appeared to have originated in the right hemisphere. In one case, however, a possible explanation for the nondominant hemispheric speech and language functions may be related to the slowgrowing neoplasm's displacement of these organizational areas.

The Wada test ${ }^{22}$ for establishment of the laterality of language function has been used in preoperative planning for decades. Pardo and Fox ${ }^{20}$ used CBF PET scanning for preoperative assessment in 11 patients with complex partial epilepsy to detect language dominance and found their results to be comparable to those of Wada testing. They concluded that CBF PET scanning provided an effective, noninvasive adjunct to the Wada test. Binder, et al., ${ }^{3}$ in a study of 22 patients with epilepsy, compared results of the Wada test and fMR imaging in which they used a singleword semantic decision task to determine language dominance. Correlation between the two tests was statistically significant and, in eight of 11 patients studied, the assessments were equivalent. In two cases the Wada test results were indeterminate, although fMR imaging provided clear evidence of hemispheric dominance. In one patient, a discrepancy between the study results occurred, and fMR imaging predicted outcome. Although only two patients in the current study (Cases 1 and 2) underwent Wada testing, concordance with PET activation studies was documented. Cortical function mapping in patients with brain tumors, however, offers several advantages over the Wada test. These include: 1) identification of functionally eloquent cortex in relationship to the tumor; 2) a noninvasive and reliable technique applicable to children as young as 3 years of age; and 3 ) the demonstration of specific speech and language functional areas compared with the nonspecific laterality of Wada tests. In view of the anatomical and functional variances of language organization, the need for exact localization of speech and language functions, in some cases, is essential for appropriate surgical management.

Preoperative FDG and/or CMET PET scanning has added dimension to our understanding of the metabolic character and behavior of brain tumors. The FDG PET studies are clearly useful to differentiate benign from malignant tumors and to predict outcome. ${ }^{1}$ The presence of increased FDG uptake has correlated with survival time in patients with malignant gliomas ${ }^{14}$ and areas of increased FDG uptake in histologically proven low-grade gliomas may be used to predict malignant evolution or degeneration of these tumors. ${ }^{5,11}$ Therefore, the combination of histopathological findings and PET data may be more predictive of outcome than the results of either modality alone. The CMET PET studies may be used to document the extent of tumor and to differentiate low-grade neoplasms. ${ }^{4}$ Both tracers, however, are useful in the prediction of histological grade and tumor progression. ${ }^{4,13}$ The results of the FDG and CMET PET studies in our cases were consistent with those of prior studies, confirming decreased FDG activity in both low-grade glial tumors. Interestingly, although they had similar neuroimaging characteristics to other low-grade tumors, both DNTs in our study demonstrated decreased metabolic activity on both 


\section{Functional brain mapping for pediatric brain tumor surgery}

FDG and CMET PET studies. This suggests a unique metabolic profile for DNTs. ${ }^{12}$

\section{Conclusions}

The cases outlined in this report support the role of PET data, including functional brain mapping, in the understanding of the metabolic character of brain tumors and preoperative planning for children who require brain tumor surgery. Areas of eloquent cortical function in relationship to tumor can be defined by coregistration of MR imaging and functional PET data. Knowledge of these higher cortical functions in the region of tumor may decrease incidences of surgical morbidity. Although we are aware of the limitations of PET scanning, including low spatial resolution and the fact that it cannot be used to rule out completely eloquent brain functions that are not activated, we believe the maps were instrumental in achieving effective surgical treatment. Functional brain mapping has the potential to alter neurosurgical approaches, maximize resections with reduced risks of neurological impairments, and to aid in the prediction of outcome.

\section{Acknowledgments}

The authors gratefully acknowledge Lang-Sheng Yun (technical assistance), Sandy Goodwin and Leslie Mullen (PET technologists), Tricia Giurlani and David Stith (radiopharmaceutical production), and Susan Fraley (manuscript preparation)

\section{References}

1. Alavi JB, Alavi A, Chawluk J, et al: Positron emission tomography in patients with glioma. A predictor of prognosis. Cancer 62:1074-1078, 1988

2. Atlas SW, Howard RS II, Maldjian J, et al: Functional magnetic resonance imaging of regional brain activity in patients with intracerebral gliomas: findings and implications for clinical management. Neurosurgery 38:329-338, 1996

3. Binder JR, Swanson SJ, Hammeke TA, et al: Determination of language dominance using functional MRI: a comparison with the Wada test. Neurology 46:978-984, 1996

4. Derlon JM, Petit-Teboué MC, Chapon F, et al: The in vivo metabolic pattern of low-grade brain gliomas: a positron emismetabolic pattern of low-grade brain gliomas: a positron emis-
sion tomographic study using ${ }^{18} \mathrm{~F}$-fluorodeoxyglucose and ${ }^{11} \mathrm{C}$ sion tomographic study using ${ }^{18} \mathrm{~F}$-fluorodeoxyglucose
L methylmethionine. Neurosurgery 40:276-288, 1997

5. De Witte O, Levivier M, Violon P, et al: Prognostic value of positron emission tomography with $\left[{ }^{18} \mathrm{~F}\right]$ Fluoro-2-deoxy-Dglucose in the low-grade glioma. Neurosurgery 39:470-477, 1996

6. Duncan JD, Moss SD, Bandy DJ, et al: Use of positron emission tomography for presurgical localization of eloquent brain areas in children with seizures. Pediatr Neurosurg 26: areas in child

7. Fox PT, Mintun MA: Noninvasive functional brain mapping by change-distribution analysis of averaged PET images of $\mathrm{H}_{2}{ }^{15} \mathrm{O}$ tissue activity. J Nucl Med 30:141-149, 1989
8. Fried I, Nenov VI, Ojemann SG, et al: Functional MR and PET imaging of rolandic and visual cortices for neurosurgical planning. J Neurosurg 83:854-861, 1995

9. Golman S, Wickler D, Damhaut P, et al: Positron emission tomography and brain tumors. Acta Neurol Belg 97:183-186, 1997

10. Haglund MM, Berger MS, Shamseldin M, et al: Cortical localization of temporal lobe language sites in patients with gliomas. Neurosurgery 34:567-576, 1994

11. Kaplan AM, Lawson MA, Bandy DJ, et al: Prognostic value of positron emission tomography in childhood low-grade astrocytomas. Ann Neurol 44:565, 1998 (Abstract)

12. Kaplan AM, Manwaring KH, Moss SD, et al: PET using $\left[{ }^{18} \mathrm{~F}\right]$ fluorodeoxyglucose and $\left[{ }^{11} \mathrm{C}\right] \mathrm{L}$-methionine to metabolically characterize dysembryoplastic neuroepithelial tumors. Ann Neurol 42:535, 1997 (Abstract)

13. Kaschten B, Stevenaert A, Sadzot B, et al: Preoperative evaluation of 54 gliomas by PET with fluorine-18-fluorodeoxyglucose and/or carbon-11-methionine. J Nucl Med 39:778-785, 1998

14. Kim CK, Alavi JB, Alavi A, et al: New grading system of cerebral gliomas using positron emission tomography with F-18 fluorodeoxyglucose. J Neurooncol 10:85-91, 1991

15. LeBlanc R, Meyer E: Functional PET scanning in the assessment of cerebral arteriovenous malformations. Case report. J Neurosurg 73:615-619, 1990

16. LeBlanc R, Meyer E, Bub D, et al: Language localization with activation positron emission tomography scanning. Neurosurgery 31:369-373, 1992

17. Mintun MA, Fox PT, Raichle ME: A highly accurate method of localizing regions of neuronal activation in the human brain with positron emission tomography. J Cereb Blood Flow Metab 9:96-103, 1996

18. Mueller WM, Yetkin FZ, Hammeke TA, et al: Functional magnetic resonance imaging mapping of the motor cortex in patients with cerebral tumors. Neurosurgery 39:515-521, 1996

19. Ojemann GA: Cortical organization of language. J Neurosci 11:2281-2287, 1991

20. Pardo JV, Fox PT: Preoperative assessment of the cerebral hemispheric dominance for language with CBF PET. Hum Brain Map 1:57-68, 1993

21. Pardo JV, Fox PT, Raichle ME: Localization of a human system for sustained attention by positron emission tomography. Nature 349:61-64, 1991

22. Wada J, Rasmussen T: Intracarotid injection of sodium amytal for the lateralization of cerebral speech dominance. Experimental and clinical observations. J Neurosurg 17:266-282, 1960

23. Woods RP, Mazziotti JC, Cherry SR: MRI PET registration with automated algorithm. J Comput Assist Tomogr 17: 536-546, 1993

Manuscript received January 3, 2000.

Accepted in final form January 3, 2000.

Address reprint requests to: Allen M. Kaplan, M.D., Neurology Department, Phoenix Children's Hospital, 909 East Brill Street, Phoenix, Arizona 85006.

This article was previously published in the Journal of Neurosurgery, Volume 91, pp 797-803, 1999. 